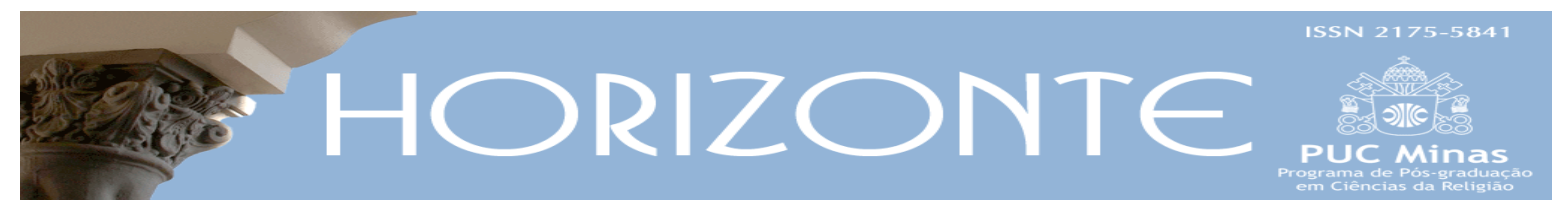

Dossiê: Ordens Religiosas Medievais: Poder e Sociedade - Articulo Originale (c) $\underset{\mathrm{EY}}{\mathrm{i}}$

DOI - 10.5752/P.2175-5841.2017v15n48p1324

\title{
La memoria e il presente. Tre sermoni su Bernardino da Siena (di Roberto Caracciolo da Lecce, di Giacomo della Marca, di Bernardino da Feltre).
}

\author{
Memory and the present. Three sermons on Bernardino da Siena (by Roberto \\ Caracciolo from Lecce, by Giacomo della Marca, by Bernardino da Feltre).
}

Maria Giuseppina Muzzarelli *

\begin{abstract}
Riassunto
Nel 1451, nel 1460 e nel 1494 tre diversi predicatori, Giacomo della Marca, Roberto Caracciolo e Bernardino da Feltre, celebrano la grandezza di Bernardino da Siena scomparso nel 1444. I sermoni dei tre appartenenti all'Osservanza francescana hanno lo scopo di mantenere la memoria di questo "secondo Francesco" e di offrire alle folle un modello. Sono tre mattoni utili per la ricostruzione della vita del Senese ma soprattutto per agire sugli uomini e sulle donne che accorrevano a sentire i tre famosi ed assai seguiti predicatori. Tutto il discorso è un impasto di ricostruzione storica e di impegno nel presente come si coglie bene soprattutto nel sermone su Bernardino da Siena di Giacomo della Marca che rende emblematicamente l'intento di usare la memoria per agire sugli uomini del suo tempo in una continuità attiva con Bernardino da Siena ma anche con Francesco.
\end{abstract}

Parole Chiave: Predicazione; Bernardino da Siena; Roberto Caracciolo; Giacomo della Marca; Bernardino da Feltre

\begin{abstract}
In 1451 , in 1460 and in 1494, three different preachers, Giacomo della Marca, Roberto Caracciolo and Bernardino da Feltre, celebrated the greatness of Bernardino da Siena who died in 1444. The sermons of the three members of the Franciscan Observance have the purpose of keeping the memory of this "second Francis" and to offer the crowds a model. The sermons are like three bricks useful for the reconstruction of Senese life, but above all for acting on the men and women who came to hear the three famous preachers. All the discourse is a mixture of historical reconstruction and commitment in the present as is best seen especially in the sermon on Bernardino da Siena by Giacomo della Marca that emblematicly makes the intent of using memory to act on men of his time in an active continuity with Bernardino da Siena but also with Francesco.
\end{abstract}

Keywords: Preaching; Bernardino da Siena; Roberto Caracciolo; Giacomo della Marca; Bernardino da Feltre

Articolo presentato il 11 luglio 2017 e approvato il 12 dicembre 2017.

* Dottore in storia medievale, laureata in Filosofia presso la Facoltà di Lettere e Filosofia dell'Università di Bologna (1973), Professoressa ordinaria di Dipartimento di Storia Culture Civiltà dell'Università di Bologna. Paese di origine: Italia. E-mail: maria.muzzarelli@unibo.it

Horizonte, Belo Horizonte, v. 15, n. 48, p. 1324-1353, out./dez. 2017 - ISSN 2175-5841 


\section{Osservazioni introduttive}

Bernardino da Siena è morto il 20 maggio $1444^{1} \mathrm{e}$ subito è iniziato il processo di canonizzazione². L'anno dopo Barnaba da Siena ha composto una vita di Bernardino seguita l'anno successiva, il 1446, dalla "Vita Bernardini Senensis" di Leonardo Benvoglienti (LÉONARD BENVOGLIENTI, 1902).

Fra il 1447 e il 1449 Giovanni da Capestrano ha scritto la "Vita Sancti Bernardini Senensis" (IOHANNEN DE CAPISTRANO, 1635, I, pp. XXVII-XL) che dunque non è la prima Vita di Bernardino ma quella che risulta aver avuto maggior rilevanza nella canonizzazione di Bernardino e verosimilmente la più diffusa e quindi nota.

Giovanni aveva vissuto a lungo con il santo ed ebbe una parte importante nelle vicende legate al processo di canonizzazione come l'aveva avuta nel difenderlo al processo in cui il Senese venne accusato di eresia per l'invenzione del trigramma IHS. Il ruolo di Giovanni, diventato procuratore dell'Osservanza nella terza e definitiva indagine processuale, fu fondamentale nell'istruzione del processo di canonizzazione e nelle indagini. La biografia fu scritta proprio negli anni della beatificazione del Senese (1447-49) con l'intenzione di rinnovare l'interesse intorno alla figura di Bernardino il cui processo di canonizzazione era rimasto bloccato per alcuni anni. Nella sua opera convergono la biografia di Benvoglienti, le testimonianze e i racconti raccolti a posteriori e soprattutto materiale di prima mano costituito dai suoi ricordi personali. Non ci occuperemo in questa sede del testo del Capestrano ma in alcuni casi faremo riferimento ad esso quale matrice comune agli autori dei tre sermoni su Bernardino da Siena a quali dedicheremo la nostra attenzione. Il testo del Capestrano non doveva servire per parlare direttamente alle folle ma da esso attinse a piene mani chi parlò alle folle compiendo delle selezioni o

\footnotetext{
${ }^{1}$ Per una sintetica biografia di Bernardino da Siena vedere ad vocem Dizionario Biografico degli Italiani, voce compilata da Raul Manselli (1967, p. 215-226). Vedere inoltre: Accademia Tudertina (1976); Domenico Maffei \& Paolo Nardi (1982) e Daniele Solvi (2014).

${ }^{2}$ Vedere Letizia Pellegrini (2009), in particolare l'introduzione della curatrice Letizia Pellegrini. Vedere anche: Ludovic Viallet (2011a, p. 420-471) e Ludovic Viallet (2011b, p. 421-434).
} 
almeno scegliendo di dare più spazio ad alcuni temi e meno ad altri. Già da quest'opera di valorizzazione e di scarto si potrebbe trarre materia per qualche ragionamento.

Nel 1451, nel 1460 e nel 1494 tre diversi predicatori di innegabili capacità comunicative hanno dunque celebrato la "magnificenza" (nel senso di "magna facere", come si dice esplicitamente nei sermoni stessi) di Bernardino da Siena. Tutti e tre appartenevano all'Osservanza Francescana e tutti si rivolgevano alle piazze per celebrarlo. In tutti e tre i casi, infatti, si tratta di sermoni che avevano lo scopo di mantenere nel pubblico la memoria di questo "secondo Francesco", di offrire alla folla radunata nelle piazze per la predicazione un modello da ammirare ma anche argomenti utili a rinforzare, attraverso la figura del Senese, l'azione che ognuno di loro, Roberto Caracciolo, Giacomo della Marca e Bernardino da Feltre stava compiendo nella città in cui predicava: Padova in due casi su tre.

Un primo elemento da mettere in rilievo riguarda il fatto che quello che si dice nei tre diversi sermoni su Bernardino da Siena è evidentemente giudicato funzionale a convincere e a commuovere le folle e viene concepito per offrire elementi di conoscenza adatti agli uditori sollecitati all'azione seguendo l'esempio del Senese.

Un secondo elemento da sottolineare è che in tutti e tre i sermoni si tracciano le linee di un percorso che parte da lontano, dallo stesso Francesco, da tutti più volte evocato (DELCORNO, 1977 e 2009, p. 159-183; MERLO, 2003), e porta al Senese per poi continuare. Proprio la continuazione appare come uno degli scopi principali all'interno di una predicazione che tutti e tre i predicatori dichiarano debba essere volta all'utile, al fare, all'ottenere frutti (MUZZARELLI, 2014)3: tratti che hanno caratterizzato l'azione ed anche la teorizzazione del Senese, come ricordano i tre predicatori, riconducendo a lui la loro forte pulsione all'azione.

${ }^{3}$ In particolare vedere Ippolita Checcoli (2014, p. 209-224). 


\section{Il sermone di Giacomo della Marca}

Il discorso più ampio, completo e più esplicitamente capace di sintesi fra la ricostruzione della vita e delle opere del Senese e l'azione sul presente, fra la memoria delle "magnificenze" di Bernardino da Siena e i temi della predicazione in corso, è quello di Giacomo Della Marca (DELCORNO, 2009)4. Giacomo è noto come efficace predicatore (PICCIAFUOCO, 1976; CANDELA, 1976 e BRACCI, 2010) ma lo sono altrettanto, se non di più, anche Roberto Caracciolo e Bernardino da Feltre. Dal confronto fra i tre sermoni che ci interessano, quello di Giacomo risulta essere il più impastato di passato e presente, di ricordo e di azione. Terremo perciò il suo sermone al centro di questo discorso che continuamente slitterà dalla predica di Roberto Caracciolo (SEVESI, 1932, p. 377-398)5 a quella di Bernardino da Feltre (VARISCHI, 1964) ${ }^{6}$ per tentare la ricostruzione del tessuto della memoria del Senese realizzato a più mani nell'arco di pochi decenni. Tale tessuto è stato fabbricato per avere una base sulla quale utilmente poggiarsi per la manutenzione del ricordo, per l'istruzione dei fedeli, per la riforma dei costumi, per il rafforzamento dell'Ordine, vale a dire per affrontare il futuro a partire dal presente, anzi dal passato. Per la composizione di tale tessuto i predicatori hanno intrecciato una serie di fili, non sempre gli stessi, tratti dalla biografia del santo e giudicati utili al proseguimento dell'azione efficace svolta dall'Osservanza7.

Per anticipare quello che verremo dicendo, nel sermone di Giacomo assumono particolare rilievo tre elementi e cioè il frequente riferimento al rapporto personale fra i due, il passaggio altrettanto continuo fra l'esperienza menzionata del Senese e l'azione in corso dello stesso Giacomo in particolare in tema di lotta alle

\footnotetext{
${ }^{4}$ Due prediche di Giacomo della Marca; Padova, 1460. In: Delcorno, 2009, p.327-377, il testo della predica è alle p. 356-377, di qui in avanti Giacomo della Marca, Sermone e predica ad honore e laude de Santo Bernardino (dalla prima riga del testo "Sermone e predica ad honore e laude de Sancto Bernardino")

${ }^{5}$ Il sermone che è di Roberto Caracciolo da Lecce e non di Michele Carcano come si legge nel titolo del saggio, è alle p. 386-398. Sulla vicenda relativa alla attribuzione del sermone vedere: Giacinto Pagnani (1954, p. 203-207); Oriani Visani (1999, p. 275-317). Di qui in avanti: Roberto Caracciolo, Sermo de Sancto Bernardino. In: SEVESI, 1932, p. 377-398.

${ }^{6}$ Sermo 82, p. 487-498. Sul Tomitano vedere: GUSLINO (2008). Di qui in avanti: Bernardino da Feltre, De sancto Bernardino, In: VARISCHI, Carlo (éd.), 1964, vol I, sermo 82, p. 487-498.

${ }^{7}$ Vedere (MIXSON \& ROEST, 2015).
} 
vanità ed infine la preminenza data all'efficace e innovativa opera omiletica del santo che ha profondamente condizionato chi è venuto dopo di lui e il modo di predicare in generale

Questi elementi erano presenti anche nel testo del Capestrano che, pur non facendo riferimento al tema parlando di Bernardino da Siena, valutò rilevante la lotta alle vanità tanto da comporre uno specifico trattatello "Degli ornamenti specie delle donne" (CAPESTRANO, 1956.) ${ }^{8}$. Giovanni operò a lungo con il Senese e nella biografia valorizzò l'aspetto fortemente innovativo, quasi rivoluzionario dell' opera di Bernardino da Siena, la sua spinta al fare e la centralità dell'omiletica. Al riguardo mi pare significativo, nell' opera del Capestrano, il racconto del giovane Bernardino al momento della peste del 1400 quando si attivò per assistere i malati ed organizzò la vita dell'ospedale di Santa Maria della Scala fino a conquistarsi la fiducia dei suoi reggitori. Nell'occasione riuscì a indurre con parole accorate e convincenti i giovani senesi a curare i malati sfidando il timore del contagio. In questo racconto si combinano efficacemente due elementi: la spinta ad agire del senese e la potenza della sua parola capace di indurre a fare (DELCORNO, 2014).

Della peste e della parte avuta dal Senese nell'assistere i malati parlano sia Roberto Caracciolo sia il Feltrino mentre nel sermone di Giacomo la peste compare a proposito della sua esperienza diretta, quando Giacomo stesso venne colpito dalla peste a Fabriano e per miracolo, grazie al Senese, riuscì a guarire.

Tornando al sermone di Giacomo, in esso, pur dedicato al Senese e dunque alla ricostruzione di vicende passate, è continuo il riferimento al presente e al personale impegno a Padova di Giacomo per moderare lussi e vanità, esperienza che si colloca al centro del suo discorso. Egli impiega il ricordo del Senese per rendere più potente l'azione che sta svolgendo. Nel caso di questo sermone è evidente l'uso della memoria per una specifica finalità, vale a dire l'utilità pratica della

\footnotetext{
8 II trattato "De usu cuiuscumque ornatus" è stato verosimilmente composto fra il 1434 e il 1438 per indicazione ricevuta nel 1434 dal vescovo di Ferrara Giovanni da Tossignano che intendeva disciplinare l'uso degli strascichi. Vedere; Maria Giuseppina Muzzarelli (1996, in particolare p. 165-210).
} 
ricostruzione delle vicende relative al Senese per la campagna di predicazione in corso. Si tratta di una predica molto lunga nella quale c'è spazio sia per il presente sia per la ricostruzione delle vicende bernardiniane utilizzate per indurre i padovani a prendere provvedimenti contro lussi e vanità.

\section{Il sermone di Roberto Caracciolo da Lecce}

Ha un taglio diverso il sermone di Roberto Caracciolo che pure condivide con Giacomo e con il Feltrino la sottolineatura dell'innovatività e della fruttuosità dell’impegno omiletico del Senese. Come Giacomo, egli ritiene che con il santo abbia avuto luogo una modificazione radicale nella predicazione (DELCORNO, 2013, p. 353) e contribuisce a riconoscere e a valorizzare agli occhi degli uditori tale svolta. Il sermone di Roberto Caracciolo, che scrive una decina d'anni prima di Giacomo, è più aderente alla vita del Santo rispetto a quello di Giacomo, più volto alla ricostruzione storica che al rafforzamento di uno specifico tema di effettiva predicazione. Uno dei tratti caratteristici della predica di Roberto è costituito dalla valorizzazione dello speciale legame con la Vergine, peraltro presente anche nei sermoni degli altri due predicatori. Il Caracciolo inoltre dà parecchio spazio a elementi biografici: i genitori del Senese, la sua infanzia, l'impegno da giovinetto al tempo della peste, la primigenia preferenza per l'eremo e il successivo ritorno alla città e relativa scelta dell'impegno attivo celebrato da Roberto in questi termini: "O benedecta illa hora, punctus et dies". Tali termini richiamano Petrarca (LOMMATZSCH, 1923, p. 675690; SCARANO, 1901, p. 250-36o, rifluito in 1971, p. 167-268.) (Canzoniere, XIII: "I’benedico il loco e 'l tempo e l'ora..."; LXI: "Benedetto sia 'l giorno, e 'l mese, et l'anno,//et la stagione, e 'l tempo, et l'ora, e 'l punto, e 'l bel paese, e 'l loco ov'io fui giunto....") e consuonano con molte indicazioni temporali della tradizione trobadorica ma anche presenti in Dante (Vita Nova XXIV, 2) e in Boccaccio (Filostrato III 83-84: "E benedico il tempo, l'anno e 'l mese, / il giorno, l'ora e 'l punto che costei / onesta, bella, leggiadra e cortese, / primieramente apparve agli occhi miei"). 
La devozione del Senese per la Vergine Maria, fortemente sottolineata dal Caracciolo, e lo stretto legame con la Vergine era tema molto presente ed insistito nella Vita di Giovanni da Capestrano, tanto da far pensare che l'autore volesse più che sottolineare inculcare l'idea della rilevanza del legame, forse per sostenere e rinforzare l'ortodossia del Senese dopo le polemiche sul trigramma di sua ideazione (HOFER, 1955, p. 136-147).

Un altro elemento, oltre a quello costituito dal legame con la Vergine, che ritorna nel sermone del Caracciolo dedicato al Senese e lo caratterizza è il ripetuto riferimento ai detrattori di Bernardino. Probabilmente negli anni più prossimi alla morte del santo erano particolarmente diffuse le resistenze a riconoscergli tutte quelle virtù che poi gli vennero attribuite. Sono elementi che lasciano scorgere tensioni intorno alla figura del santo. Il riferimento all'accusa di eresia è presente anche nel sermone Feltrino ma in maniera più cursoria.

Roberto ha riferito dell'accusa di eresia pazientemente sopportata dal Senese parlando di Bernardino-luna mentre Giacomo lo fa parlando di Bernardino-sole, là dove si sofferma sulle persecuzioni che dovette subire per l'invenzione del Nome di Gesù. Anche a questo riguardo Giacomo fa cenno alla sua personale esperienza dicendo che il Senese venne a consiglio da lui prima di andare in Concistoro per difendersi e "lo spirito sancto intrò in la sua lingua, feze tanto e tanto seppe dire che ogniuno rimaxe confuso et satisfato" (GIACOMO DELLA MARCA, 2009, p. 374): ancora una volta la parola giocò un ruolo determinante e rese Bernardino vincitore dopo essere stato martire come lo era stato Francesco che "mandò a catare l soldano solo perché lo avesse caxone de marturizarlo" (GIACOMO DELLA MARCA, 2009, p. 374).

Tanto la sottolineatura del legame con la Vergine come la chiamata in causa dei detrattori da parte del Caracciolo consentono di cogliere, dal suo sermone, un clima di tensione intorno al Senese del quale Roberto ricorda la vasta cultura ("omnibus doctoribus moderni et theologie et iuri canonico diligenter studebat") cosa che Giacomo non fa. Caracciolo fa riferimento alle principali opere del senese 
("tria pulcra opera compilavit") ed anche alla sua capacità di dilettare gli uditori. Quest'ultimo tema appare anche nel sermone di Giacomo che parla di un modo di predicare del Senese attraente e soave: "tanto era suave quello suo modo et inventione" e "ogniuno de ogni de ogni qualità ne portava a caxa (GIACOMO DELLA MARCA, 2009, p. 359): al centro, sebbene in misura e forma diversa, di tutti i tre sermoni ma soprattutto di quello di Roberto si colloca dunque la fruttuosità del modo di predicare del Senese.

Roberto Caracciolo dà molto spazio ai miracoli "quasi 500 fuerunt", mentre per il Feltrino erano 400 quando invece Giacomo parla di "più de tre milia" in un'evidente discordanza e dice di avere visto gli effetti di alcuni di questi miracoli con i suoi occhi, il che solo in parte si trova anche nel sermone di Giacomo. Quest'ultimo ha il vantaggio, rispetto al Caracciolo, di essere stato personalmente miracolato dal Senese, il che rafforza quel legame diretto di cui si è parlato come elemento che caratterizza il sermone di Giacomo rispetto agli altri due.

Nella predica del Caracciolo sono presenti in filigrana più città: Perugia divisa al suo interno, L’Aquila dove fu vista la stella sul capo di Bernardino, Siena dove visse il santo, Roma dove all'inizio quasi nessuno andava ad ascoltarlo ma che poi "odorem sentire coepit". C'è un'Italia che richiedeva un'urgente opera di riforma dei costumi ma non c'è l'urgenza del presente come in Giacomo che inframezza il racconto relativo al Senese con parole e raccomandazioni rivolte ai padovani nel pieno della sua azione di modificazione del loro "modus vivendi".

\section{Il sermone di Bernardino da Feltre}

Quanto al sermone di Bernardino da Feltre, esso prende avvio rappresentando in maniera diretta la piena condivisione dell'urgenza del fare del senese e il valore dell'efficacia dela predicazione: il Feltrino dichiara infatti di non volersi perdere in inutili esordi, "star ad exordire sicut homines mundani" come 
"citarista che in principio fa una bella recerchata" ma di volersi mettere "direttamente a tavola" senza lavarsi le mani ("ma ponam me ad mensam sine lavare manus”) (BERNARDINO DA FELTRE, 1964, p. 487). Dunque teorizza e pratica il passaggio diretto all'azione, al fare che nel caso specifico consisteva nell' illustrare il "magnificus qui magna facit. Si magnificentia reperitur, reperitur ergo in magna facientibus". I fatti al centro ma "Quid fecit...?” Ecco la risposta: ha saputo trionfare contro la superbia: "tute le grandeze mundi se le ha messe sub pedibus...non bisogna querere triunfar pompatice de mundo" (BERNARDINO DA FELTRE, 1964, p. 488). Requisito e accompagnamento del suo trionfo contro la superbia è l'umiltà del Senese e la sua obbedienza. Nell'esaltazione del "magnificus qui magna facit" torna più volte il termine "magnus" esteso anche a Francesco: "Sanctus Bernardinus et sanctus Franciscus sic fuere magni quanto plura dexpexerunt” (BERNARDINO DA FELTRE, 1964, p. 491). Bernardino è “magnus” perché incorruttibile ma anche per la sua vittoria sulla carne: morì infatti vergine. Grande pericolo "quando se va reto a tante delicatezze e tanti relaxamenti, a vivere voluptuosi: chè non fa tanta batalia come el mundo cum questi balli e canti” (BERNARDINO DA FELTRE, 1964, p. 492).

Il riferimento diretto a Francesco, parlando di Bernardino da Siena, si ritrova in tutti e tre i sermoni che stiamo considerando. Il Caracciolo dice che il Senese fu inviato dalla Vergine assieme a Vincenzo Ferrer [i due grandi predicatori sono accostati per la prima volta nella biografia di Vincent Ferrer che Pietro Ranzano scrisse nel 1455 per la canonizzazione del santo domenicano (DELCORNO, 2006, p. 7-38.)] per rimediare al fatto che il mondo era in maximo periculo: "malefitias, incantationes, sortilegia, divinationes, periuria” (ROBERTO CARACCIOLO, 1932, p. 388). Ciò dopo che la Vergine aveva mandato un paio di secoli prima Francesco e Domenico per contrastare le brutture mondane che stavano per indurre Dio a distruggere il mondo. Al loro tempo Francesco e Domenico fecero cessare tanti mali con la loro dottrina, vita ed esortazione tanto che la sentenza fu revocata. Poi le cose tornarono come prima e ciò ha reso necessario l'invio appunto di Bernardino da Siena e di Vincenzo Ferrer. Giacomo della Marca a sua volta collega il Senese a 
Francesco ricordando le persecuzioni patite dal Senese che connette al martirio cercato da Francesco presso il soldano.

Bernardino ha pazientemente sopportato e dato prova di grande umiltà mettendosi sotto i piedi, riferisce il Feltrino, tutte le "grandeze mundi". I trionfi (e qui fa riferimento ai Trionfi di Petrarca) non sono quelli mondani ma "iste mundus va per bagatelle" (BERNARDINO DA FELTRE, 1964, p. 489). Bernardino trionfò contro la superbia con la sua umiltà fatta di ubbidienza e di fortezza. Segue un discorso sulla povertà e sulla ricchezza:

Povertà è nulla havere. Chi possede è posseduto [...]. Ille qui plus vult ascender oportet quod plus ponat sub pedibus...Quanto plus mundum despicit tanbto plus elevatus...O le richeze.... chi ha la granda bocca pizzol boccon non li basta...homo ita deditus divitiis non ha fiato in corpo (p.491) ...Sanctus Bernardinus et sanctus Franciscus sic fuere magni quanto plura dexpexerunt....O Sanctus bernardinus quantum fuit agnus! Sprevit omnia quando fuit Mediolani, che dux Franciscus donavit quelle bacine plene ducato rum [...] sprevit omnia istius mundi, omnes cupiditates (BERNARDINO DA FELTRE, 1964, p. 490).

Il termine "magno" (da cui "magna facere", "magnificentia" e "magnificare") torna a proposito sia di Francesco sia del Senese "sic fuere magni quanto plura dexpexerunt" (BERNARDINO DA FELTRE, 1964, p. 491).

Quando Giacomo aveva parlato dell'umiltà di Bernardino aveva introdotto un lungo discorso sui suoi tempi e sulle vanità femminili concluso con una raccomandazione ai padovani: "siati soliciti al vostro ben comune" "notate e schrivete molto bene che frate Jacopo de la Marcha si l'à dito adì 20 de mazo 1460 in Padoa"... Ora a caxa"

A caxa comporta trattare il tema di Bernardino-sole. Dunque anche il discorso sull'umiltà del Senese era servito a Giacomo per rendere più efficace la sua azione a Padova. 
Ricorda Caracciolo che Bernardino nacque l'8 settembre, giorno della Natività della Vergine. Fu battezzato in quel giorno e sempre nello stesso giorno a 22 anni prese l'abito di Minore (tre significative coincidenze). L'anno dopo nello stesso giorno fece la professione e l'anno dopo ancora cantò la prima messa e sempre nello stesso giorno cominciò a predicare (ROBERTO CARACCIOLO, 1932, p. 389). La Vergine volle rendere visibile la sua predilezione e all'Aquila quando predicava davanti a 50.000 persone e teneva un sermone sulla vergine Maria "visa fuit quaedam stella” (ROBERTO CARACCIOLO, 1932, p. 389)99.

Anche Giacomo della Marca fa riferimento alla speciale relazione che legava Bernardino alla Vergine parlando degli anni che precedettero la sua entrata nell'Ordine (GIACOMO DELLA MARCA, 2009, p. 373). Il medesimo legame privilegiato è indicato dal Feltrino secondo il quale il Senese "Fuit impetratus et venit per manus Marie quia omes gratie per manus eius transeunte $t$ misit in mundum” (BERNARDINO DA FELTRE, 1964, p. 493) e aggiunge che l'Italia ne aveva un gran bisogno e che proprio in considerazione del "gran bisogno" vennero inviati Vincenzo Ferrer e Bernardino da Siena. Di questo duplice invio aveva parlato, come già riferito, anche il Caracciolo.

Anche il Feltrino affronta il tema della relazione speciale del Senese con la Vergine che lo mandò "in mundum", assieme a Vincenzo, ben consapevole di quanto l'Italia e la Spagna avessero bisogno di loro: invii mirati, diciamo così.

Della vita del santo il Feltrino ricorda l'assistenza agli appestati e il fatto che non voleva farsi predicatore per via della sua voce roca ma che un globo igneo gli purificò la lingua tanto da poter parlare fino a 7 ore conferendogli una tale eloquenza che "non errabat pur d'una parolla” (BERNARDINO DA FELTRE, 1964, p. 494). A Bernardino egli riconosce tutte le prerogative del grande oratore: "bel corpo, la lingua cossì diserta, quella voce cossì sonora, tanto compunctiva che passava li cori

${ }_{9}^{9}$ Sul tema mariano nelle vite di Bernardino da Siena: Daniele Solvi (2007/2011, p. 371-390). 
e medulle". Sono così indirettamente indicate le caratteristiche che il predicatore efficace deve avere: potenza fisica, proprietà nel parlare e voce adeguata. Tutto ciò era presente nel Senese del quale il Feltrino sottolinea la innata, forse ispirata capacità e la partecipazione intensa in grado di farsi parola efficace. Rappresentazione plastica di ciò è offerta dall'immagine di Bernardino da Siena "infuocato": "El ha el fogo adosso, et ponit adosso auditori bus suis; verba sua procedunt a medullis cordis: carbon morto non potest" (BERNARDINO DA FELTRE, 1964, p. 496).

Per il Feltrino Bernardino da Siena era un fuoco e trasmetteva fuoco: un giovane che si rivolse a lui dicendogli che avrebbe voluto servire Dio ma si sentiva il cuore tutto freddo venne prese da Bernardino da Siena per i capelli e "totus fuit ignitus amore Dei” e divenne frate minore e fu un buon frate. Era un fuoco che si diffondeva e procurava effetti come in effetti accadde in numerose città: Milano, Bergamo, Brescia e tante altre (BERNARDINO DA FELTRE, 1964, p. 496). Era un fuoco che proveniva dal cuore e insieme dalla Vergine che a L'Aquila, mentre egli predicava, volle mostrarsi presente facendogli comparire una stella sul capo: "Quando predicavit Aquile de Corona Virginis, tanto ben cantò che la sua manza se fece alla fenestra. Che crede tu che fusse quella stella che apparui super caput, nisi illa Maris stella?” (BERNARDINO DA FELTRE, 1964, p. 495).

Non arte ma dono alla base della sua abilità oratoria: "Como pulcram artem habeti? ...Nullam artem habeo" salgo sul pulpito "ad honorem Dei et salutem animarum...Quando el fogo caza la bombarda, lassela andar" (BERNARDINO DA FELTRE, 1964, p. 495).

Come Giacomo, anche il Feltrino riconosce che il santo ha rinnovato la vita dei cristiani combattendo usurai e concubini: queste sono magnificenze, azioni importanti ("magna") fatte dal santo analoghe a quelle elencate da Giovanni da Capestrano nella sua biografia: sedare le risse, comporre le inimicizie, ottenere restituzioni, eliminare i giochi, disciplinare i lussi. A tutto ciò il Capestrano ha fato 
riferimento testimoniando direttamente le grandi cose fatte dal Senese al quale attribuiva importanti risultati ben resi dall'indicazione di numeri strabilianti: prima di Bernardino i frati erano 130, dopo di lui "ultra 4.000" mentre i conventi passarono da 20 a 230 quanti divennero alla morte del santo e a 270 al momento in cui Giovanni scriveva la sua biografia (CAPISTRANO, 1635). Niente del genere compare nei nostri tre sermoni.

A mezzo secolo dalla morte di Bernardino da Siena si è costruita, osserva il Feltrino, una catena osservante a partire dal Senese che "nunziavit Michele, Spoletinum, Capistranum e Giacomo della Marca”. Bernardino da Siena, afferma il Feltrino, "rexit nos" "ista povera familia" che si caratterizza, a partire dalla sua "buona guida" non dall'estendersi "in subtili scientia" ma dal "parlar affocamente", ispirati da Dio come Francesco "quod Sanctus Franciscus non posuit verbum de suo, sed sicut rivelavi Christus" (BERNARDINO DA FELTRE, 1964, p. 496). Tanto il Senese come il Feltrino appartengono alla "Religio" denominata Serafica che non si estende in sottigliezze scientifiche ("in subtili scientia" e il Feltrino, come il Senese dice di voler "sempre parlar affocamente" in una evidente sintesi fra i due Bernardini.

\section{Predicare per fare: la "grande reprensione"}

Questo, a grandi linee, relativamente ai tre sermoni dei quali ora intendo esaminare più nel dettaglio alcuni aspetti sempre tenendo al centro quello di Giacomo della Marca. Giacomo, come Roberto, in avvio del sermone dedicato al Senese menziona il passo dell'Ecclesiaste "Erit sichut stela mattutina et quasi luna plena...et quasi sol refulgiens" e immediatamente collega il discorso su Bernardino al presente facendo riferimento alla magnifica città di Padova, dove stava predicando, come luogo in cui "si magnifica et honora" il santo nei confronti del quale denuncia la carenza delle sue capacità di celebrarlo (GIACOMO DELLA MARCA, 2009, p. 356). Anche il Caracciolo peraltro si era detto inadeguato a tessere 
le lodi di Bernardino (ROBERTO CARACCIOLO, 1932, p. 386). Pur sentendosi inadeguato, Giacomo avverte il bisogno, anzi l'urgenza di celebrare il Senese a partire da quello che conosce. Fin dall'inizio del sermone e più volte Giacomo fa riferimento alla relazione personale ed intensa con il Senese "me fosti compagnio e maestro" e “de incurabile infermità mi resanasti” (GIACOMO DELLA MARCA, 2009, p. 356357).

Anche il Feltrino farà riferimento, ma in termini generici, a rapporti con il Senese che è "stato cum noi a manzar e bever" (BERNARDINO DA FELTRE, 1964, p. 496) dicendo inoltre del Senese che ha annunciato Michele (Carcano), Spoletino (Cherubino da Spoleto), Giovanni da Capistrano, Giacomo della Marca "chè ipse rexit nos" ma è Giacomo ad attingere ripetutamente a un bagaglio di ricordi personali e a spendere questa relazione speciale come testimonianza utile e insieme fonte di credito. Relazione speciale intrattenuta quando Bernardino era vivo ma anche da morto per via del miracolo compiuto dal santo che da defunto guarì Giacomo. La celebrazione di Giacomo inizia con la dichiarazione di questo debito di riconoscenza che è anche la attestazione di un miracolo: si avvia cioè con un formidabile legame che rafforza il discorso e l'azione di Giacomo.

Roberto Caracciolo introduce una sua diretta testimonianza parlando dei miracoli compiuti da Bernardino: "in civitate Spoleti vidi quamdam dominam...", "quemdam iuvenem oculis meis vidi..." "quaedam domina vocata perla quam vidi..." (ROBERTO CARACCIOLO, 1932, p. 396).

Stella bella, lucida, graziosa ma soprattutto annunciatrice, scrive Giacomo. Stella che manifesta il giorno e guida chi si mette in cammino, che dà luce e riscalda. Nelle oscure tenebre (i tempi in cui gli capitò di vivere) Bernardino-stella ha annunciato la comparsa del sole predicando il santo nome di Gesù che è stato dipinto su palazzi e scolpito in più forme (GIACOMO DELLA MARCA, 2009, p.357) ${ }^{10}$. Il giorno che annunciò e che poi ebbe inizio fu il muovo ed efficace modo di predicare,

10"fo anonciatore de questo dolcissimo Nome, lo quale prima non era in uxo né in devotione apresso le persone". 
capace di fruttificare, che con lui prese avvio. Prima di Bernardino si predicava "dopo dixnare" e quasi nessuno andava alla predica. Lui invece predicava alla mattina e indusse vecchi e giovani ad andarvi: in questo senso fu stella e guida. Fu un vero lume che arrecò luce nel campo della predicazione in quattro modi: con invenzioni, ornata eloquenza, bei gesti e modi ma anche con minacce e aspre riprensioni. Prima di lui le prediche sembravano dispute e molti tornavano a casa confusi mentre Bernardino "acatò uno modo atrativo a le audientie" tanto che predicando quattro o cinque ore non sembrava passata che mezz'ora e "ognuno de ogni quallità ne portava a caxa” (GIACOMO DELLA MARCA, 2009, p.359). Al riguardo il Feltrino dice che quando il Santo predicava "videbant ignem egredi et andar adosso a le persone, che le affocava" e che non ricorreva a inutili sottigliezze diversamente da quelli che predicavano "zanze" come si legge in Dante "canto 29 Paradisi" (BERNARDINO DA FELTRE, 1964, p.487). Il Feltrino, lo abbiamo anticipato, riconduceva direttamente a Dio la straordinaria capacità oratoria del Senese. Dio gli purificò la lingua tanto da renderlo capace di parlare per 5, 6 o 7 ore, sempre Dio gli diede una adeguata corporatura, voce sonora e il talento di "affocare" le persone. L'alba annunciata dalla stella Bernardino è per Giacomo quella delle prediche nuovo stile, non più adornate di eloquenza. Prima di lui "quando intravano in qualche sotil materia predicavano più a sì che ad altri...e niente se pigliava". Le sue prediche erano adorne di molti belli gesti e modi che pareva di essere in paradiso oppure all'inferno, sembrava che quella anima sancta avesse fate tute le arte... Prima di lui quando i predicatori montavano in pergolo "stavano durri e quasi in tuta la predicatione se voltavano resonando in modo de oratione" (BERNARDINO DA FELTRE, 1964, p.487). Del modo di predicare ha parlato alle folle direttamente il Senese in una predica sul tema (BERNARDINO DA SIENA, 1989, p. 141-173) ${ }^{11}$.

La nuova forma di predicazione annunciata e praticata dal senese prevedeva anche "grande reprensione" e Giacomo della Marca riferisce, nella sua predica sul Senese, di avere attaccato con vigore a Padova le vanità femminili mescolando

${ }^{11}$ Vedere: M.G.Muzzarelli (2005, in partic. cap.l, p. 15-93). 
ancora una volta il passato con il presente: "E certo cossì se vol fare, o padoani mie': in questa Quaresema ....sempre ve ho reprendù e maximamente queste done con queste sue gran corna in cavo, che pareano proprio de queste sele todesche e de queste sue gran code.” (GIACOMO DELLA MARCA, 2009, p.360)

La decisione dell'attacco non scoraggiò il pubblico: "e volete bene e non stadi de vegnire a la mia predica....E questo è advegnudo perché non ho guardado in faza, e dicovi sul volto li vostri deffecti e mancamenti." (GIACOMO DELLA MARCA, 2009, p.350) $)^{12}$

Giacomo, come dice ai padovani, intende seguire le orme del grande innovatore Bernardino (GIACOMO DELLA MARCA, 2009, p.361) ${ }^{13}$ che cambiò il modo di predicare e conseguentemente anche l'atteggiamento degli uditori. Questi ultimi prima di lui restavano tiepidi, anzi freddi e "quando le persone stavano a messa, la più parte, levato lo corpo de Cristo, subito loro se lavaveno in piè" mentre con Bernardino "stano firmi in zonichioni fina che lo sacerdote rezeve lo corpo e sangue dil Signiore" ; con lui il popolo intrecciava una sorta di dialogo, dopo essere stato indotto a frequentare la messa e la predicazione:"che prima era come uno mondo morto"(GIACOMO DELLA MARCA, 2009, p.361).

Parlando di Bernardino-stella Giacomo affronta in maniera diretta la questione della predicazione efficace che è al centro di tutti e tre i sermoni, e fa scivolare il discorso da Bernardino a sé medesimo, dai frutti attesi ed ottenuti dal Senese a quelli che a lui più importano: "E certo così se vol fare, o padoani mei in questa Quarexema [...] sempre ve ho reprendù e maximamente queste done con queste sue gran corne in cavo [...].”(GIACOMO DELLA MARCA, 2009, p.360)

Il nesso fra le lodi del Senese e la lotta che Giacomo sta conducendo a Padova contro le vanità si fa stretto quando egli affronta il tema Bernardino-luna. La luna

\footnotetext{
${ }^{12}$ Se io invece, aggiunge Giacomo, "ve fosse andado con bellini e luxenge" non vedreste l'ora che io partissi.

13 Prima di Bernardino i predicatori cercavano la benevolenza del popoli, "non se curava de andar driedo a queste reprensione". Bernardino è stato fuori e dentro all'Osservanza e dopo di lui chi voleva ben predicare "à tolto lo stillo, la forma de questo glorioxo santo". Vedere: C. Delcorno (2009, p. 291-326).
} 
illumina, e il discorso torna sulla luce che il Senese ha portato con la sua predicazione, ma la luna è anche rotonda, umida e vacua. In merito alla sfericità Giacomo dice che Bernardino: "volse esser in tuto tondo, aver una bona scientia e non andare su per le frasche che in conscentia tante e tante sotigeze sono piuttosto nocive che utill" (GIACOMO DELLA MARCA, 2009, p.362)

In riferimento all'umidità Giacomo dice che Bernardino "foe in tuto humido zoè che senpre sechretamente lui pianzeva la Passione de Iesu" e che fu umido nella sua predicazione per le molte lacrime sparse dal popolo che lo seguiva. In alcune città 30-40.000 persone assistettero alla sua predica ${ }^{14}$ ed era un stupore sentire la voce e i pianti del popolo che commosse e convertì inducendo usurai e meretrici a cambiar vita (GIACOMO DELLA MARCA, 2009, p.363):

Quante baratarie per lui desfate...Quanti incantanti e libri de incantatori...dade carte tavoleiri "de ogni cossa insenbre ne fexe fare monti altissimi, insembre con le dreze de le femene". Che a quel tempo pareveno che avesse una gran cesta on capo et al presente queste done le àno abassade in mezzo che non sono quaxi niente e da li ladi àno alzade doe grandissime corne.

A questo punto e su questo tema Bernardino predicatore impegnato contro lussi e vanità si sovrappone a Giacomo che predica sullo stesso tema e nel parlare di copricapi riprovevoli testimonia un cambiamento della moda: se al tempo della predicazione di Bernardino in Campo de'Fiori le vanesie portavano in testa un copricapo che pareva una gran cesta, al tempo di Giacomo, cioè una trentina d'anni dopo il copricapo si era abbassato in mezzo e molto alzato ai lati a mo' di sella o di corna ed il fenomeno è comprovato dall'iconografia (GNIGNERA, 2010).

A partire da questo spunto Giacomo introduce un intervento sui suoi tempi e sulla volubilità delle donne per smascherare la quale esorta i giovani a portare in giro

${ }^{14}$ Sulla folla che assisteva alle prediche vedere: Bruni (2003, pp. 182-192); Muzzarelli (2005, p. 158-166). 
un asino con in testa "qualche fantasia nova" e farlo vedere a tutti. Si dichiara disposto a non predicare mai più se in capo a otto giorni:

le done de questa tera quella fantaxia che voi averì fata in cavo a lo asino loro done la comenzerà a levare e portare. Sì che, a preposito, quante diversse sise (mode) e mainiere de tenpo in tenpo, e tuta è stà opera del diavolo da lo inferno, che a quel tempo de san Bernardino che io dico, le dreze vostre parevano grande zeste piene de zeliexe, e lo diavolo non potea tropo bene sentare a destro suo. E lui se conzigniò de farvele fare per altra mainiera, in modo de selle todesche, azò che lui possa meglio repossare.

(GIACOMO DELLA MARCA, 2009, p.363)

L'aggettivo umido fa anche riferimento alla capacità del Senese di far intenerire la gente "che lasasse simel vanità". Ancora una volta il tema sottolineato è quello della predicazione efficace e in particolare efficace contro le vanità.

Trattando della luna vacua Giacomo parla di Bernardino come vacuo nel senso di privo di ambizioni, "e non come fano alchuni ...che sono tanto gonfiati, che chi li toccasse e ponzesse con una gusela ge toria fuori l'aqua de le ongie vinti pedi” (GIACOMO DELLA MARCA, 2009, p.365).

L'umile Bernardino cucinava in grembiule per gli altri frati, si dedicava a opere manuali (sapeva fare belle scodelle per la minestra e bellissimi zoccoli) e si occupava anche dell'orto: richiesto di potare, pur non avendo esperienza riuscì bene anche in quello diversamente da me, dice Giacomo, che pur andai con lui nell'orto.

L'impasto fra passato e presente, la connessione-identificazione fra Bernardino da Siena e Giacomo realizzata sul tema delle vanità viene riproposta da Giacomo anche quando parla dell'umiltà del Senese. Giacomo dice che Bernardino, alle cui prediche milanesi parteciparono fino a 60.000 persone, non volle mai nessuno al seguito e lo stesso voglio fare io, aggiunge Giacomo che dichiara di non apprezzare: "chi me vien driedo, chi me tira la capa, chi me tocha i zocholi, chi con le forbici cerca di taglairmi il mantello"....Chi credete voi che io sia? Se me avete amor, padoani, teneteme amor caritativo...” (GIACOMO DELLA MARCA, 2009, p.366) 
Giacomo riferisce di essere richiesto a Vicenza, a Verona, a Treviso ma che non fatica a separarsi dai padovani considerati gli ottimi frutti della sua predicazione

però che io ho visto che con grande diligentia avete ottenuto nel Conseglio vostro quello che zà gran tempo ve ho predicado zoè che queste manege de pano d'oro che portano le vostre done, e tanti zuponi de arzento e manege da done fodrate de arzento e dui e tri vestimenti de sa ha la dona de talle che non ha pan a manzare, che zerto èp una cossa da ruinare e rivare de impoverire e far lo zodio molto ben richo. (GIACOMO DELLA MARCA, 2009, p.366-367)

Giacomo osserva che in un anno non si sono fatti che tre matrimoni e che quindi è stata "una santa cossa" disciplinare i lussi come lui aveva suggerito ${ }^{15}$ ed aggiunge che renderà noto ovunque quello che hanno fatto i padovani che dovranno proseguire nell'opera intrapresa: "E metete anche parte e leze a li artexani che non è onesto che loro dibiano vestire egualmente e homeni e done a paro di vuoi citadini. Ancora provedete a le dote grande...Poi finalmente de le code e de le dreze”. (GIACOMO DELLA MARCA, 2009, p.367).

Prima di riprendere il discorso su Bernardino ("a caxa”), dopo l'ampia digressione sulla lotta alle vanità in corso a Padova, Giacomo esorta i padovani: "siati soliciti al vostro ben comune...e notate e schrivete molto bene che frate jacopo de la Marcha si l'à dito adì 20 de mazo 1460 in Padoa”... Ora a caxa. (GIACOMO DELLA MARCA, 2009, p.368).

Il tema dell'umiltà del Senese ha dunque offerto a Giacomo lo spunto per un inserto sulle vanità dei suoi contemporanei e sulla necessità di porre un freno a tanti sprechi. Più che un innesto è il miglior modo di celebrare Bernardino facendo quello che lui ha insegnato a fare e continuando il suo impegno con fatti e non solo con parole. Giacomo sovrappone al discorso sul Senese quello sulla lotta alle vanità che lo avvicina ed accomuna a Bernardino con ciò realizzando più che asserendo una continuità rafforzata anche dai numerosi i ricordi personali di Bernardino proposti

\footnotetext{
${ }^{15}$ Si veda: Antonio Bonardi (1910, in partic. p. 147-150). Vedere: Paola Monacchia \& Maria Grazia Nico (2013, pp.279-300).
} 
da Giacomo. Uno dei ricordi riguarda il loro camminare, insieme a un terzo, nelles Marche: avevano fame e sete e nessuno li sfamava, quando finalmente raggiunsero una fontana e Giacomo stava per avventarsi sull'acqua ma Bernardino consigliò prudenza e suggerì dove trovare un po' di pane ed in effetti lo trovarono. Un'altra testimonianza personale attesta l'essere Bernardino privo di ogni egoismo in quanto prestò graziosamente a Giacomo i suoi sermoni (GIACOMO DELLA MARCA, 2009, p.365) ma anche l'essere privo di ogni superbia e infatti, ricorda Giacomo, il Senese cucinava per i frati "dolcemente e allegramente" (GIACOMO DELLA MARCA, 2009, p.365-366).

Prima di Giacomo già Roberto Caracciolo aveva comincia il suo sermone sul Senese con i quattro raggi di Bernardino "stella radians" dicendo che egli fu mandato sulla terra dalla Vergine "ratione utilitatis" e stabilendo due legami diretti: uno fra Bernardino e la Vergine (come si trova anche negli altri sermoni) e l'altro con Francesco. Al tempo di Francesco e di Domenico Dio voleva distruggere il mondo a causa dei troppi peccati e la Vergine fece opera di intercessione contando su Francesco e Domenico. Grazie alla loro azione cessare in effetti molti mali e la sentenza fu revocata. Passato l'effetto immediato dell'azione di Francesco e di Domenico, continua il Caracciolo, il mondo ripiombò "in maximo periculo: malefitias, incantationes, sortilegia, divinationes, periuria" (ROBERTO CARACCIOLO, 1932, p. 388). Di nuovo la Vergine intervenne e chiese al figlio: "noli currere ad iudicium... fac unum qui lingua sua mundo nunzie sua scelera et sic evadat tuum flagellum". Anche il Senese come Francesco risulta "grande riparatore" di disastri e il mondo fu ancora una volta salvato dalla parola, vale a dire dalla predicazione di Bernardino in Italia e di Vincenzo Ferrer fuori d'Italia (DELCORNO, 2006, p.7-38).

Anche il Feltrino, alcuni anni dopo, farà riferimento alla coppia BernardinoVincenzo. In Italia nessuno fu più utile di Bernardino che intervenne per far cessare blasfemie e restituire le usure. A Perugia c'erano grandi divisioni che Bernardino pacificò e lo stesso a Roma. 
Nel discorso del Caracciolo, ove ricorre due volte di seguito il concetto di "magnam utilitatem", si stabilisce una diretta relazione fra Francesco e Bernardino. Anche il Feltrino fa riferimento in più casi a Francesco e a proposito del disprezzo delle ricchezze dice: "Sanctus Bernardinus et Sanctus Franciscus sic fuere magni quanto plura dexpexerunt” (BERNARDINO DA FELTRE, 1964, p.487).

Una parte non breve del sermone del Feltrino è dedicata alla predicazione del Senese capace di smorzare o risolvere discordie e di chiarire le idee anziché confonderle (qui che si colloca la già menzionata citazione del canto 29 del Paradiso di Dante). Il discorso era volto a invitare a distinguere, a partire dal caso esemplare del senese fino ad arrivare allo stesso feltrino, tra predicazione capziosa, troppo sottile, inefficace e predicazione illuminante ed utile. Come asserito dallo stesso Bernardino da Siena nella sua predica sulla predica tenuta nel 1427 sul Campo de' Fiori (MUZZARELLI, 2005), se il predicatore vuole convincere ed ottenere risultati deve gettare una rete che "non vol essere tropo né sutile né tropo grossa" per ricorrere alle parole del Feltrino (BERNARDINO DA FELTRE, 1964, p.495) mentre il Senese più che di rete aveva parlato di ami e di esche. Bernardino da Feltre riprende dunque il tema del parlare "chiarozzo chiarozzo" dicendo che "non canta bene se dal cor non vene”. Il Senese cantò tanto bene, quando predicò a L'Aquila sulla corona della Vergine, che la Vergine si materializzo sotto forma di una stella che apparve sopra al suo capo (BERNARDINO DA FELTRE, 1964, p.495) ${ }^{16}$. La stella per il Feltrino è il segno della approvazione di Maria, della giusta via intrapresa dal Senese con la scelta di farsi capire privilegiando l'utile.

Caracciolo, diversamente da Giacomo della Marca (ma anche del Feltrino che nell'economia di un sermone relativamente breve non offre molto spazio alle vicende biografiche) dedica parte del suo sermone al racconto della famiglia di Bernardino e dei primi anni di vita del santo. Bernardino fu l'unico figlio di due devote persone che morirono presto. Seguirono gli anni con la zia che si accorse di una passione di

${ }^{16}$ Ne parla anche Roberto Caracciolo (1932, p. 389). 
Bernardino per qualcuno che si rivelò essere la Vergine. Giacomo riferisce molto brevemente elementi della biografia del Senese tra cui la nascita del forte suo legame con la Vergine Maria e la sua entrata nell'Ordine e sostiene che fu lo stesso Senese a raccontargli personalmente "come fue lo suo prencipio ed ogni cossa" (GIACOMO DELLA MARCA, 2009, p. 372-373).

Caracciolo, come Giacomo, attribuisce importanza al "fare" di Bernardino: giovanissimo, quasi sapesse che lo aspettava l'officium praedicatoris volle prima fare che insegnare esercitandosi in opere di pietà e di carità. Esemplare l'operato di Bernardino in tempo di peste a Siena nel 1400: non solo si impegnò direttamente davanti a un fuggi fuggi generalizzato ma riuscì a convincere altri a farlo (ROBERTO CARACCIOLO, 2006, p. 391-392): la sua parola (come più tardi la sua predicazione) seppe indurre a fare.

Un elemento che caratterizza il discorso di Roberto Caracciolo su Bernardino è il riferimento ai detrattori che lasciano capire come intorno a Bernardino e più in generale intorno all'Osservanza Francescana ci fosse favore ma anche avversione farlo (ROBERTO CARACCIOLO, 2009, p. 392). Procedendo nel racconto degli anni giovanili del Senese, Caracciolo riferisce che dopo quattro mesi di assistenza agli appestati Bernardino si ammalò "in habitu seculari” e che poi entrò nella religione rinunciando, non abdicando al mondo. "Come ti sei comportato quando sei entrato nella religione?" si chiede Roberto Caracciolo che cerca una risposta nei sermoni dello stesso Bernardino. Qui si situa un nuovo riferimento ai detrattori, in questo caso avversari dell'Ordine. Inizialmente Bernardino decise di lasciare tutto e andare a vivere in solitudine. Sopportò più che potè la fame mangiando qualche radice $\mathrm{e}$ bevendo acqua per riuscire a degluttirle ma capì che non era questa la via: tornò torna in città andò al monastero di $\mathrm{S}$. Francesco ed entrò nella regola. "O benedecta illa hora, punctus et dies quando sanctus Bernardinus habitum suscepit" dice Roberto a proposito del momento in cui entrò nell'Ordine farlo (ROBERTO CARACCIOLO, 2009, p. 393). In quel momento scelse di operare fra la gente, di rendersi utile facendo splendere il raggio della sua feconda predicazione. 
Roberto Caracciolo giunge alla magnificazione della feconda predicazione del senese parlando di Bernardino-luna dai quattro splendori, il primo dei quali è la "fructuosa praedicatio" (gli altri 3 sono: "profunda humiliatio", "patiens supportatio", "studiosa exercitatio"). La predicazione per andare a buon fine deve docere, flectere, delectare (ROBERTO CARACCIOLO, 2009, p. 393). La realizzazione di queste tre finalità è evidente in Bernardino e Roberto si chiede chi mai più di lui abbia insegnato tanto utilmente al popolo. Bernardino "Predicabat secundum Auditorum capacitatem pro salutis sue via, adeo quod quilibet fructum capiebat”. (ROBERTO CARACCIOLO, 2009, p. 393)17

Quanto all'umiltà, Bernardino fu umile “in core, ore et opere”. L'umiltà non consiste nel lavare scodelle o nel porsi in infimo posto bensì nel rinunciare ad onori per rimanere umili. Sono molti coloro che vestono abiti umili per offrire l'immagine del disprezzo del secolo ma se assunti a dignità la accettano. Bernardino ha rinunciato alla dignità episcopale alla quale fu eletto a Urbino, a Siena, a Ferrara dichiarandosene indegno. Accettò per obbedienza di essere vicario dell'Ordine ma ben presto vi rinunciò (ROBERTO CARACCIOLO, 2009, p. 393-394).

Sopportò pazientemente malanni e persecuzioni. Roberto, a differenza da Giacomo e Bernardino da Feltre fa riferimento, come si è detto, a detrattori e accusatori del Senese e in particolare all'accusa di eresia che sopportò con mansuetudine e pazienza tale che indussero Martino $\mathrm{V}$ a riaffidargli l'incarico di predicare. Sapendolo accusato di eresia molti non andarono a sentirlo ma lui perseverò e alla fine la gente accorse alle sue prediche. I detrattori pare che sostenessero la limitatezza del suo repertorio di prediche, una quarantina: "O fatui, si unus 40 sermones, bene potest 50 et centum addiscere" (ROBERTO CARACCIOLO, 2009, p. 394).

\footnotetext{
${ }^{17}$ Dalla sua capacità di adattamento derivarono grandi esiti: per ben 30 anni flexit animos audientium e non perseverò certo "spe pecunie".
} 
Diversamente da quanto sostenuto dai detrattori il Senese studiò ininterrottamente per 40 anni. Il tema dello studio non è presente in Giacomo mentre Roberto fa esplicito rimando alle opere composte da Bernardino da Siena.

\section{Per concludere: la morte di Bernardino}

Roberto conclude parlando di Bernardino-sole rilucente "ex felici consumatione, ex miraculorum perpetratione, ex gloriosa canonizatione, ex letitia totius mundi”. Tutti e tre i sermoni finiscono riferendo della morte e dei miracoli del Senese che si spense all'età di 64 anni. Morì a L'Aquila città ricca e divisa al suo interno tanto da essere quotidianamente teatro di omicidi o almeno così, riferisce Roberto, diceva Giovanni da Capestrano. Dio volle aiutare la città facendo morire a L'Aquila Bernardino: appena morto le campane cominciarono a suonare. Tutti e tre i predicatori ricordano il fatto che prima di morire volle essere spogliato ed essere deposto in terra. Giacomo dice che "lui instesso pigiava pian piano con la mano destra la sua capa e faxeva de signio in modo che lo voleva che loro la tirasseno fuora. E cossì loro lo fexeno”. (GIACOMO DELLA MARCA, 2009, p. 376)

Sempre Giacomo dice che era di età decrepita e che le forze gli erano venute a mancare: "aveva perso le potencie, ni altro per quello signialle voleva dire se non che voleva morire nudo cossì come lui nassette nudo". (GIACOMO DELLA MARCA, 2009, p. 376)

Il Feltrino racconta che: "Quando morti proximus fuit se voleva pur tirar zoso de la letera, e morir in terra, voleva pur tirar le man forade le maniche, chè voleva morir nudo in terra; sed non fuit permissus a Fratribus”. (BERNARDINO DA FELTRE, 1964, p. 487)

Alla sua morte seguirono, nel racconto di tutti e tre i predicatori, molti miracoli. Caracciolo e il Feltrino ne parlano alla fine della loro predica dopo aver riferito dela morte del Senese mentre Giacomo parla dei miracoli prima di riferire 
degli ultimi istanti del santo. Parla di due ore come tempo necessario per riferire tutti i miracoli (GIACOMO DELLA MARCA, 2009, p. 375). Su questi ultimi Giacomo si diffonde a proposito della settima proprietà del sole che "è utille a molte cose": ricorre di nuovo il concetto di utilità che assieme a quello di fruttuosità si collocano al centro della ricostruzione di Giacomo ma non solo della sua. Come il sole produce mille buoni frutti così Bernardino che ha realizzato oltre 3.00o miracoli per parlare solo di quelli compiuti "in morte e dopo la morte" a partire da quello di cui godette lo stesso Giacomo (GIACOMO DELLA MARCA, 2009, p. 396). Anche in tema di miracoli compare nel racconto di Roberto un riferimento a detrattori: "O detractor, te intellige, quia forte dicis, quod aliquando miracula facta sunt per malos nomine”. (ROBERTO CARACCIOLO, 1932, 396)

Ci vollero 4 ore, aggiunge Roberto, per elencare i suoi 500 miracoli e di uno di essi offre testimonianza personale: colpito a Fabriano dalla peste Giacomo recuperò la salute grazie a un miracolo del Senese. Altri miracoli dichiara di averli visti con i suoi occhi. Giacomo della Marca affronta il tema dei miracoli parlando di Bernardino sole rilucente, che genera e distrugge. Bernardino-sole ha prodotto grandissimi frutti, dal fondare ospedali all'abbattere cattive usanze. Tra i frutti vanno annoverati gli oltre 3.000 miracoli a lui attribuiti. E qui si inserisce la sua diretta testimonianza: al tempo della sua morte mi trovavo in un'altra città ed ero infermo, riferisce Giacomo ai padovani, braccia e mani mi tremavano. Papa Nicola mi voleva mandare in Alemagna e non credeva alla mia malattia allora andai dal papa che vedendomi si convinse. Tornando indietro passai da L’Aquila per rivedere il mio "gratioxo compagnio" mi fu aperta la cassa nella quale lui giaceva e pareva fresco come fosse vivo. Mi misi a piangere e cercai di abbracciarlo "in segnio de amore che li avevo portato in vita". La mattina seguente mi ritrovai sano come voi mi vedete (GIACOMO DELLA MARCA, 2009, p.369-371).

Sempre Giacomo riferisce che a L'Aquila, dove un incredibile numero di stampelle testimonia le innumerevoli grazie ottenute, si sostiene che almeno 12 
morti siano stati da lui resuscitati (GIACOMO DELLA MARCA, 2009, p.371-372) ${ }^{18}$. Rientra in un certo senso nel tema dei miracoli anche il fatto sottolineato da Giacomo che non si trova un altro santo canonizzato così presto e una così vasta e spontanea devozione con tanto di chiese fabbricate in suo nome e processioni e offerte, il tutto in soli 3 anni dopo la sua canonizzazione (GIACOMO DELLA MARCA, 2009, p.369375). Tra le "preeminentie" del Senese ricordate da Giacomo nell'ultima parte del suo sermone vi è l'apparizione della stella a L'Aquila mentre predicava "e lui se obstupì tuto e quasi più non predicò” (GIACOMO DELLA MARCA, 2009, p.369376). Morì il giorno dell'Ascensione (20 marzo 1445) e da lì a 8 giorni gli uscì sangue vivo dal naso cosa che, secondo i medici è: "quaxi inpossibele. Lo qual sangue io Girardo, che questo ò sscritto, viti a frate Zoane da Capestrano in certo banbaxo in uno vaxo de vetro in San Francesco in padoa, co li mei ochi propri el vidi”. (GIACOMO DELLA MARCA, 2009, p.377).

Il racconto di Giacomo era cominciato con un riferimento alla "magnifica citade de Padoa" e finisce con una sua testimonianza diretta e con un riferimento all'attualità, a quella ampolla venerata a Padova. Finisce con la raccomandazione ai padovani di implorare grazie da Bernardino "che non dubito che largamente ve le concederà et abiatillo in somma riverentia et devotione" (GIACOMO DELLA MARCA, 2009, p.371) in una sorta di personalizzazione del discorso, in una utile attualizzazione, in un impasto di ricostruzione storica e di impegno nel presente che è la cifra caratteristica del sermone su Bernardino da Siena di Giacomo della Marca ma anche l'emblema del modo di usare, non solo da parte di Giacomo, la memoria per agire sul presente.

\footnotetext{
${ }^{18}$ Giacomo dettaglia sul caso di un garzone finito sotto la ruota di un mulino e resuscitato da Bernardino e aggiunge: "quanti retrati, quanti arsiradi, quanti ziechi, quanti zopi, quanti de ogni infermità".
} 


\section{RIFERIMENTI BIBLIOGRAFICI:}

ACCADEMIA TUDERTINA. Bernardino predicatore nella società del suo tempo. Atti del XVI Convegno internazionale di studi, 9-12 ottobre 1975. Todi: Accademia Tudertina, 1976.

BERNARDINO DA FELTRE. In: VARISCHI, Carlo (éd.). Sermoni del b. Bernardino Tomitano da Feltre nella redazione di Bernardino Bulgarino da Brescia. 3 vol. Milano: Vita e Pensiero, 1964.

BERNARDINO DA SIENA. Prediche volgari sul Campo di Siena 1427. In: DELCORNO, Carlo (éd.). Milano: Rusconi, 1989.

BONARDI, Antonio. Il lusso di altri tempi in Padova. In: Miscellanea di Storia veneta, s.III, vol. II, 1910, p. 147-150.

BRACCI, Silvano. San Giacomo della Marca: divino predicatore. Cascine Vica: Elledici, 2010.

BRUNI, Francesco. La città divisa. Le parti e il bene comune da Dante a Guicciardini. Bologna: il Mulino, 2003.

CANDELA, Silvestro, S. Giacomo della marca nel V centenario della morte. Napoli: Collana di studi Giacomiani, 1976.

CHECCOLI, Ippolita. Biographies of the Franciscan Observance: Text Narrating the Deeds. In: MUZZARELLI, Maria Giuseppina (éd.). From Words to Deeds. The Effectiveness of Preaching in the Late Middle Ages. Turnhout: Brepols 2014, p. 209-224.

DELCORNO, Carlo. “Quasi quidam cantus”. Studi sulla predicazione medievale. Firenze: Olschki, 2009, p. 159-183.

DELCORNO, Carlo. Da Vincent Ferrer a Bernardino da Siena. Il rinnovamento della predicazione alla fine del Medio Evo. In: HODEL Paul-Bernard; MORENZONI, Franco. Mirificus Praedicatori. A l'Occasione du sixième centenaire du passage de Vincent Ferrier en Pays romand, Actes du colloque d'Estavayer-le.Lac, 7-9 octobre 2004. Roma: Monumenta Ordinis Fratrum Praedicatorum, Dissertationes historicae XXXII, Istituto Domenicano, 2006, p. 7-38.

DELCORNO, Carlo. L'Osservanza francescana e il rinnovamento della predicazione. In: I Frati Osservanti e la società in Italia nel secolo XV Atti del XL Convegno internazionale Assisi-Perugia, 11-13 ottobre 2012. Spoleto: Cisam, 2013, p. 3-53.

DELCORNO, Carlo. Le origini. Francesco d'Assisi. In: Francesco d'Assisi e francescanesimo dal 1216 al 1226. Atti del IV Convegno internazionale di Assisi. Assisi: S.Maria degli Angeli-Porziuncola, 1977. 
DELCORNO, Carlo. Modelli retorici e narrativi da Bernardino da Siena a Giacomo della Marca. In: DELCORNO, Carlo (éd.); BAFFETTI, Giovanni (éd.); FORNI, Giorgio (éd.); SERVENTI, Silvia (éd.); VISANI, Oriana, (éd.). “Quasi quidam cantus”. Studi sulla predicazione medievale. Firenze: Olschki 2009, p. 291-326.

DELCORNO, Pietro. La molteplice funzione politica di un episodio agiografico: il servizio di Bernardino da Siena all'Ospedale della Scala durante la peste. In: Ordens Religiosas na Idade Média: Concepções de Poder e Modelos de Sociedade (séc. XII-XV) - Congresso Internacional, 26-29 May 2014, Universidade Federal de Minas Gerais - Belo Horizonte.

GIACOMO DELLA MARCA. Sermone e predica ad honore e laude de Sancto Bernardino. In: DELCORNO, Carlo. "Quasi quidam cantus". Studi sulla predicazione medievale, a cura di G. Baffetti, G. Forni, S. Serventi, O. Visani. Firenze: Olschi, 2009, p, 356-377. [in appendice al saggio di C. Delcorno, Due prediche di Giacomo della Marca (Padova 1460), ivi, p. 327-377]

GIOVANNI DA CAPESTRANO. Degli ornamenti specie delle donne. In: CHIAPPINI ANICETO (éd.). Siena: Edizioni Cantagalli, 1956.

GNIGNERA, Elisabetta. I soperchi ornamenti: copricapi e acconciature femminili nell'Italia del Quattrocento. Siena: Protagon, 2010.

GUSLINO, Bernardino. La vita del beato Bernardino da Feltre. In: CHECCOLI, Ippolita (éd.). Bologna: Editrice Compositori, 2008.

HOFER, G. Giovanni da Capestrano: una vita spesa nella lotta per la riforma della Chiesa. Roma: S. Bernardino: 1955.

IOHANNEN DE CAPISTRANO. Vita Sancti Bernardino Senensis per B. Iohannem a Capistrano conscripta. In: DE LA HAYE, Jean (éd.). Sancti Bernardini Senensis opera omnia. Paris: Sumptibus Dionysii Moreav, 1635, I, pp. XXVII-XL.

LÉONARD BENVOGLIENTI. Vie de S. Bernardin de Sienne par Léonard Benvoglienti. In: FRANCISCUS VAN ORTROY (éd.). Analecta Bollandiana, t. 21, 1902, p. 53-80.

LOMMATZSCH, Erhard. Benedetto sia il giorno e 'l mese e l'anno. In: Zeitschrift für romanische Philologie, XLIII (1923), p. 675-690.

MAFFEI, Domenico (éd.); NARDI, Paolo (éd.). Atti del simposio internazionale Cateriniano-Bernardiniano. Siena 17-20 aprile 1980. Siena, 1982.

MANSELLI, Raul. Bernardino da Siena. In: Dizionario Biografico degli Italiani, vol. 9, Roma: Istituto dell'Enciclopedia Italiana, 1967, p. 215-226.

MERLO, Grado Giovanni. Nel nome di San Francesco. Storia dei frati Minori e del francescanesimo sino agli inizi del XVI secolo. Milano: EFR, 2003. 
MIXSON, James D (éd.); ROEST, Bert (éd.). A Companion to Observant Reform in the Late Middle Ages and Beyond. Leiden: Brill, 2015.

MONACCHIA, Paola; NICO, Maria Grazia. Gli Osservanti e la legislazione comunale in Umbria nel secolo XV. In: I Frati Osservanti e la società in Italia, Spoleto, 2013, p. 279-300.

MUZZARELLI, Maria Giuseppina (éd.). From Words to Deeds. The Effectiveness of Preaching in the Late Middle Ages. Turnhout: Brepols 2014.

MUZZARELLI, Maria Giuseppina. Gli inganni delle apparenze: disciplina di vesti e ornamenti alla fine del medioevo. Torino: Scriptorium 1996.

MUZZARELLI, Maria Giuseppina. Pescatori di uomini: predicatori e piazze alla fine del Medioevo, Bologna: Il Mulino, 2005.

PAGNANI, Giacinto. Un discorso in lode di Bernardino da Siena recitato da Roberto Caracciolo, falsamente attribuito al b. Michele Carcano da Milano, in Archivum Franciscanum Historicum, $\mathrm{n}^{\circ}$. 47, 1954, p. 203-207.

PELLEGRINI, Letizia (éd.). Il processo di canonizzazione di Bernardino da Siena (1445-1450). Grottaferrata: Ad Claras Acquas, 2009.

PICCIAFUOCO, Umberto. S. Giacomo della Marca (1393-1476): uomo di cultura, apostolo, operatore sociale, taumaturgo del sec. XV. Monteprandone: Santuario di S. Giacomo: Convento di S. Maria delle Grazie, 1976.

ROBERTO CARACCIOLO. Sermo de Sancto Bernardino. In: SEVESI, Paolo Maria. Un sermone inedito del b .Michele Carcano su S. Bernardino da Siena. In: Collectanea Franciscana, a. II, fasc. III, luglio 1932, p. 377-398.

SCARANO, Nicola. Fonti provenzali e italiane della lirica petrarchesca. In: Studi di filologia romanza, VIII (1901) p. 250-360. Rifluito in Francesco Petrarca, SCARANO, Isotta (éd.). Campobasso: Editrice I. Scarano, 1971, p. 167-268.

SEVESI, Paolo Maria. Un sermone inedito del b .Michele Carcano su S. Bernardino da Siena. In: Collectanea Franciscana, a. II, fasc. III, luglio 1932, p. 377-398.

SOLVI, Daniele (éd.). L’agiografia su Bernardino Santo (1450-146o). Firenze: Siemel Edizioni del Galluzzo, 2014.

SOLVI, Daniele. Bernardino da Siena, una santità mariana? In: BARTOLOMEI ROMAGNOLI, Alessandra (éd.) \& FREZZA, Fortunato (éd.). Amicitiae sensibus. Studi in onore di don Mario Sensi. Bollettino storico della città di Foligno, $\mathrm{n}^{\mathrm{os}} \cdot \mathbf{3 1}-34$ (2007-2011), p. 371-390.

VARISCHI, Carlo (éd.). Sermoni del b. Bernardino Tomitano da Feltre nella redazione di Bernardino Bulgarino da Brescia. 3 vol. Milano: Vita e Pensiero, 1964. 
VIALLET, Ludovic. Il processo di canonizzazione di Bernardino da Siena (1445-1450). In: Frate Francesco, $n^{\circ}$. 77, 2011a, p. 420-471.

VIALLET, Ludovic. L'Observance” franciscaine à l'epoque de la canonisation de Bernardin. Un état des lieux. In: Frate Francesco, nº. 77, 2011b, p. 421-434.

VISANI, Oriana, Roberto Caracciolo e i sermonari del secondo Quattrocento. In: Franciscana, $\mathrm{n}^{0} .1$, 1999, p. 275-317. 